\title{
Power, Subcarrier Allocation and Relay Selection with Interference Managements for OFDM-Based Cognitive Heterogeneous Networks
}

\author{
Ban Khalid Ammar ${ }^{1}$, Hayder Jawad Albattat ${ }^{1}$, Ahmed Ghanim Wadday ${ }^{1}$ and Mohannad A.M. Al-Ja'afari ${ }^{2}$ \\ ${ }^{I}$ Communication Techniques Eng. Dept, Engineering Technical College/Najaf, Al-Furat Al-Awsat Technical University, Najaf, Iraq \\ ${ }^{2}$ Electronics and Communication Techniques Dept, Al-Najaf Technical Institute, Al-Furat Al-Awsat Technical University, Najaf, Iraq.
}

[Corresponding Emails: coj.bancom@atu.edu.iq, hayder.albattat@atu.edu.iq,ahmadghw@hotmail.com and inj.muh@atu.edu.iq]

\begin{abstract}
Heterogeneous systems merging a different wireless networks to provide a maximum system capacity and to improve spectrum efficiency. In the Heterogeneous systems the problem of Resources Allocation (RA) and interference management considered as important challenges to maximize a total system capacity. An Orthogonal Frequency Division Multiplexing (OFDM) cognitive radio network is proposed in this paper to a group of small cells assisted by amplify and forward AF two-way relays, with perfect sensing. The RA and relay selection proposed in this paper as a formulation problem, to maximize the total transmission rate for small cell networks within one macro cell. The genetic algorithm GA used as optimization technique to solve this optimization problem. The results showed the ability of this algorithm to effectively determine the frequency and power values that distributed over 256 levels. To solve the problem of cross-tier interference from macro cell base station to small cell users, a theoretical analysis has been performed to calculate it and which included in calculation the total capacity according to Shannon's theory. All equations have been derived and theoretically calculated in this paper, in addition to getting higher transmission rate.
\end{abstract}

Keywords - Heterogeneous Networks; cognitive small cell; two-way AF relay; power allocation; subcarrier allocation; relay selection matrix; genetic algorithm.

\section{INTRODUCTION}

According to the increasing demand for spectrum band due to the development of communication technology the capacity improving, radio resources allocation and interference management are considered the most important challenges for this development. Heterogeneous Networks (HetNet) consist of different wireless networks it is promising to improve the total capacity, increasing the coverage area and enhance the spectrum efficiency. HetNet typically consist of one macro cell with large radius and a high transmission power that included small cells with small radius and low transmission power [1], [2]. From the concept of cognitive radio networks, allow a group of small cells to use the license spectrum owned by macro cell, with a dynamic spectrum access by spectrum sensing technique with hierarchical access model that preventing small cells to harmful interference the macro cell under interference threshold constraints, this is the simplest definition of heterogeneous networks [3]. To increase the coverage area between small cells and to help small cell users $S U s$ communicate with each other it is possible to propose the addition relay technique to small cells within the macro cell
[4-6]. Due to the advantages of subcarrier allocation and to limited the inter-carrier interference from orthogonal

subcarriers, OFDM proposed as modulation technique based on cognitive small cells assisted by relay nodes in HetNet [7]. In the related work for small cells (femto cell, micro cell, pico cell, ...) with macrocell heterogeneous network. The proposed heterogeneous model in [8] downlink transmission, using an OFDMA as a multiple access technique to limit the cross-tier and allocate a group of sub channels to different users, same idea of cognitive radio, dual decomposition method used for power allocation and sub channels allocation. A new dynamic spectrum allocation method proposed in [9] for hybrid cognitive femto cell, they used dual decomposition method to maximize the total downlink transmission rate and solved the problems of power allocation and sub channel allocation. In [10] the optimal power allocated solved by using a Lagrange method they proposed a MIMO femto cell with MIMO pico cell using the same frequency band with macro cell. In [11] two activities problems the power and sub channel allocation solved by using a game theory to maximize the uplink transmission rate between a macro 


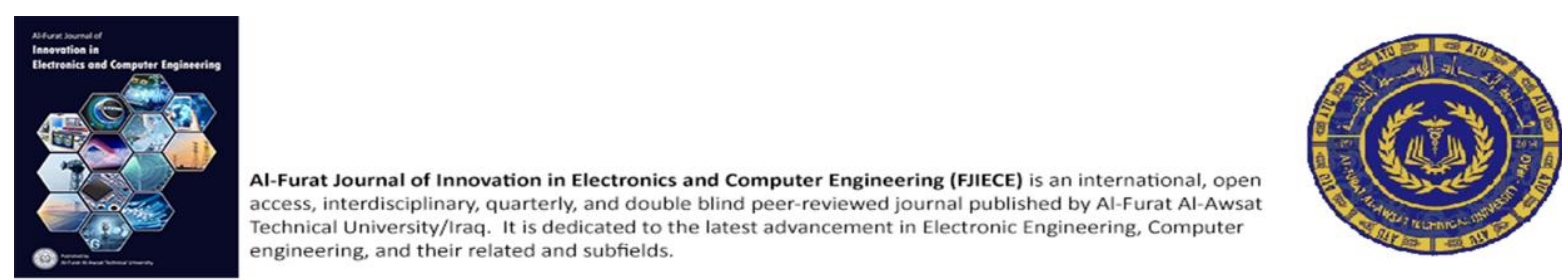

cell and multiple femto cells under the two-tier interference and delay for femto cell users. For power allocation problem in [12] solved by using Proportional-Integral (PI) controller (feedback closed loop) for OFDM femto cell with two-tiers.

In this paper, the resources allocation (power allocation and subcarrier allocation) are proposed for cognitive-based small cells and AF two-way relay nodes, under the maximum transmission power constraint for the small cell users, and the interference power (from small cells to

macro cell) constraint; and the cross-tier interference (from macro cell base station to small cell users) are simultaneously considered. The objective function is to maximization the total OFDM-transmission rate between the small cell users assisted by AF two-way relay nodes.

\section{SYSTEM MODEL}

In this paper, a multiple small cells are deployment, where cognitive HetNet is considered and consist of one macro cell, multiple-small cells and multiple-relay nodes.

Macro cell is defined as (primary network) and small cells with relay nodes are defined as (secondary networks). The set of relay nodes is defined as $\mathcal{K}=\{1, \ldots, \mathrm{k}, \ldots \mathrm{K}\}$ and the set of small cell users is defined as $\mathcal{S}=\{S U 1, S U 2, \ldots$, $S U n$ \} with the notice that (n: represents total number of small cells). The total licensed frequency band used by primary network (macro cell) can be divided into smaller parts or a specific numbers of sub-carriers that are equal to $\mathrm{N}$ subcarriers, the set of subcarriers denoted as $\mathcal{N}=\{1,2, \ldots, \mathrm{n}, \ldots, \mathrm{N}\}$. The set of subcarriers occupied by primary network and defined as $\mathrm{N}_{\mathrm{p}}=\{1, \ldots, 1, \ldots, \mathrm{L}\}$, small cell users perfectly sensing the spectrum band and detecting the unused subcarriers, and with underlay spectrum access way. The small cell users and relay nodes are accessing the spectrum and allocating the vacant subcarriers defined as $\mathrm{N}_{\mathrm{s}}=\{1, \ldots, \mathrm{m}, \ldots, \mathrm{M}\}$. OFDM technique is adopted for the transmission mechanism for the macro cell, small cells and relay nodes. Figure 1 shows in general the overall proposed system model.

The implemented system shown in Fig. 2, consist of one macro cell (primary network) with one macro cell base station (MBS) and one macro cell user pair (sender MU-s and destination MU-d). It also consist of two small cells base station (SBS1) and (SBS2).

Each small cell has one small cell user, $S U_{1}$ and $S U_{2}$, to communicate with each other through two relay nodes R1 and R2 in two-way protocol and half-duplex mode to exchange the messages between the SUs.

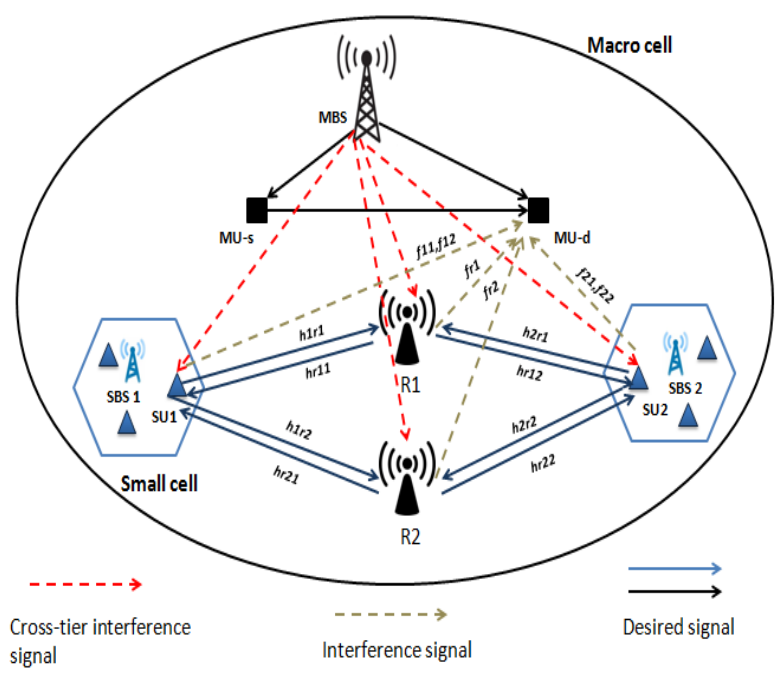

Fig.2: Implemented model

The total spectrum band is divided into 6 subcarriers $\mathrm{N}=$ $\{1,2,3,4,5,6\}$; two subcarriers are occupied by macro cell users $N_{p}=\{1,2\}$, and the other unused subcarriers are used by secondary network $N_{s}=\{3,4,5,6\}$, (where these numbers represent the indicator of subcarriers). All system definitions listed in the Table-I.

\section{TABLE-I: SYSTEM DEFINITIONS}

\begin{tabular}{|c|c|}
\hline $\mathbf{A}$ & $\begin{array}{l}\text { two dimensions matrix represent location of }\langle i, j\rangle \text { pairs, } \\
\mathrm{A}=1 \text { if }\langle i \text { for } \mathrm{MA}\rangle \text { and }\langle j \text { for } \mathrm{BC}\rangle\end{array}$ \\
\hline B & $\begin{array}{l}\text { three dimension matrix represent the subcarrier pairs } \\
\text { allocated to } K \text { relay }\end{array}$ \\
\hline $\mathcal{K}$ & Identify the number of relay nodes $\{1, \ldots, k, \ldots, \mathrm{K}\}$ \\
\hline $\mathbf{N}$ & Total Bandwidth divided into $N$ subcarrier \\
\hline$N_{p}$ & $\begin{array}{l}\text { no. of subcarrier used by macro cell (primary) user } N_{p} \epsilon \\
\mathcal{N} \\
N_{p}=\{1, \ldots, l, \ldots, \mathrm{L}\}\end{array}$ \\
\hline$N_{s}$ & $\begin{array}{l}\text { no. of subcarrier used by small cell (secondary) network } \\
N_{S} \in \mathcal{N}, N_{S}=\{1, \ldots, m, \ldots, \mathrm{M}\}\end{array}$ \\
\hline$i$ & subcarrier for MA phase from (SUs) to Relays, $i \in N_{S}$ \\
\hline$j$ & subcarrier for BC phase from relays to (SUs),$j \in N_{S}$ \\
\hline$x_{\text {si }}$ & $\begin{array}{l}\text { Represent the symbols transmitted by SUs on sub-carrier } \\
i\end{array}$ \\
\hline$p_{\text {si }}$ & $\begin{array}{l}\text { Power allocation to small cell users when sending to } k^{t h} \\
\text { relay in MA phase. }\end{array}$ \\
\hline $\boldsymbol{h}_{\boldsymbol{n}} \boldsymbol{r}_{\boldsymbol{k}}$ & $\begin{array}{l}\text { Represent the channel-gains when small cell users } \\
\text { sending to } k^{t h} \text { relay in MA phase }\end{array}$ \\
\hline$h \boldsymbol{r}_{\boldsymbol{k n}}$ & $\begin{array}{l}\text { Represent the channel-gains from } k \text {-relay to small cell } \\
\text { users in BC phase }\end{array}$ \\
\hline
\end{tabular}




\section{A. Rate analysis for Secondary Network}

To analyze the rate of secondary networks with the AF two-way relays, it takes two phases to exchange the signals between the $S U s$. The transmission through relays divided into two phases to complete the total transmission: the $S U s$ transmit simultaneously to the selected relay in MA phase on subcarrier $i$, then the selected relay amplifies the received signal with amplification factor $\left(A_{f}\right)$ and broadcasts it to SUs in broadcast phase (BC) on subcarrier $j$ [13].

1. Multi Access (MA) phase: the SUs users transmit signals in the same time to the $k$-relay on the allocated subcarrier $(i)$. The signal received at the $k^{\text {th }}$ relay is [13]:

$$
y_{i}^{k}=\sqrt{p_{1 i}} h_{1} r_{k} x_{1 i}+\sqrt{p_{2 i}} h_{2} r_{k} x_{2 i}+\theta_{i}^{k}+z_{i}^{k}
$$

Where $\theta_{i}^{k}$ is a thermal noise power of AWGN at the relay $k$ which caused by SUs on sub-carrier $i$, and it is same in all receivers (assuming the mean equals to zero $\mu=0$ and the variance $\left.\sigma^{2}=0.01\right)$ which are defined as $\left(\mu, \sigma^{2}\right)$, then $\theta_{i}^{k^{2}}=\sigma^{2}$. And $Z_{i}^{k}$ Cross-tier interference from MBS with each signal transmits from SUs to $k^{t h}$ relay in MA on subcarrier $i$.

Assuming the total noise power from SUs to relay nodes is denoted by a symbol $n r_{i}^{k}$, and calculated in eq.(2)is given by.

$$
n r_{i}^{k}=\sigma^{2}+Z_{i}^{k}
$$

2. Broad Cast $(B C)$ phase: the $k^{\text {th }}$ relay then amplifies the received signal with amplification factor $A_{f}$ [13], [15] and forwards this signal on the subcarrier $j$, assuming the expectation operator of $\mathrm{E}\left[\left|x_{1 i}\right|^{2}\right]=1, \mathrm{E}\left[\left|x_{2 i}\right|^{2}\right]=1$ is denoted by E[.] [14].

$$
A_{f}=\frac{1}{\sqrt{p_{1 i}\left|h_{1} r_{k}\right|^{2}+p_{2 i}\left|h_{2} r_{k}\right|^{2}+\left(n r_{i}^{k}\right)^{2}}}
$$

The received signal at $\mathrm{SU}_{1}, \mathrm{SU}_{2}$ from $k^{\text {th }}$ relay (broadcast signal) [13], [15] on subcarrier $j$ denoted: $y_{1 j}$, $y_{2 j}$, respectively.

$y_{1 j}=A_{f} \sqrt{p_{r j}} h r_{1 k} y_{i}^{k}+\theta_{j}^{k}+z_{j}^{k}$

\footnotetext{
Inter-user Interference

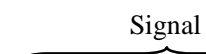

$y_{1 j}=$

$A_{f} \sqrt{p_{r j}} h r_{1 k} \sqrt{p_{1 i}} h_{1} r_{k} x_{1 i}+$

$A_{f} \sqrt{p_{r j}} h r_{1 k} \sqrt{p_{2 i}} h_{2} r_{k} x_{2 i}+A_{f} \sqrt{p_{r j}} h r_{1 k} n r_{i}^{k}+n_{1 j}^{k}$

(5)
}

Noise $y_{2 j}=A_{f} \sqrt{p_{r j}} h r_{2 k} y_{i}^{k}+\theta_{j}^{k}+z_{2 j}^{k}$

$$
\begin{aligned}
& \text { Signal }
\end{aligned}
$$

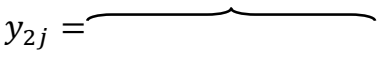

$$
\begin{aligned}
& A_{f \sqrt{p_{r j}}} h r_{2 k} \sqrt{p_{1 i}} h_{1} r_{k} x_{1 i}+ \\
& A_{f} \sqrt{p_{r j}} h r_{2 k} \sqrt{p_{2 i}} h_{2} r_{k} x_{2 i}+A_{f} \sqrt{p_{r j}} h r_{2 k} n r_{i}^{k}+n_{2 j}^{k} \\
& \text { (7) } \\
& \text { Noise }
\end{aligned}
$$

Where, $Z_{1 j}^{k}$ and $Z_{2 j}^{k}$ are the cross-tier noises assumed in this paper, from MBS with each signal broadcasted from $k^{\text {th }}$ relay to $\mathrm{SU}_{1}, \mathrm{SU}_{2}$, respectively in $\mathrm{BC}$ phase on subcarrier $j$, on the assumption that the total noise signal assumed at $\mathrm{SU}_{1}$ is denoted by $n_{1 j}^{k}$ and $\mathrm{SU}_{2}$ denoted by $n_{2 j}^{k}$, and can be calculated in (8) and (9), respectively:

$n_{1 j}^{k}=\sigma^{2}+z_{1 j}^{k}$
$n_{2 j}^{k}=\sigma^{2}+z_{2 j}^{k}$

With perfect channel state information that leads to the inter-user interference part in (5) and (7) which can be eliminated using channel estimation [14], [15], then the signal to cross-tier interference plus noise ratio ( SINR) can be calculated as:

$$
\begin{aligned}
& \operatorname{SINR}_{1}=\frac{A_{f}^{2} p_{r j}\left|h r_{1 k}\right|^{2} p_{2 i}\left|h_{2} r_{k}\right|^{2}}{A_{f}^{2} p_{r j}\left|h r_{1 k}\right|^{2} n r_{i}^{k^{2}}+n_{1 j}^{k^{2}}} \\
& \operatorname{SINR}_{2}=\frac{A_{f}^{2} p_{r j}\left|h r_{2 k}\right|^{2} p_{1 i}\left|h_{1} r_{k}\right|^{2}}{A_{f}^{2} p_{r j}\left|h r_{2 k}\right|^{2} n r_{i}^{k^{2}}+n_{2 j}^{k^{2}}}
\end{aligned}
$$

By dividing (10) by $\left(A_{f}^{2} n r_{i}^{k^{2}} n_{1 j}^{k^{2}}\right)$, (11) by $\left(A_{f}^{2} n r_{i}^{k^{2}} n_{2 j}^{k}{ }^{2}\right)$, and substituting this equation:

$\frac{1}{A_{f}^{2}}=p_{1 i}\left|h_{1} r_{k}\right|^{2}+p_{2 i}\left|h_{2} r_{k}\right|^{2}+\left(n r_{i}^{k}\right)^{2}$, the result equal to:

$$
\begin{gathered}
\operatorname{SINR}_{1}=\frac{\frac{A_{f}^{2}}{A_{f}{ }^{2}} p_{r j} \frac{\left|h r_{1 k}\right|^{2}}{n_{1 j}^{k^{2}}} p_{2 i} \frac{\left|h_{2} r_{k}\right|^{2}}{n r_{i}^{k^{2}}}}{\frac{A_{f}^{2}}{A_{f}{ }^{2}} p_{r j} \frac{\left|h r_{1 k}\right|^{2}}{n_{1 j}^{k^{2}}} \frac{n r_{i}^{k^{2}}}{n r_{i}^{k^{2}}}+\frac{n_{1 j}^{k^{2}}}{A_{f}{ }^{2} n_{1 j}^{k^{2}} n r_{i}^{k^{2}}}} \\
=\frac{P_{r j} H R_{1 k} P_{2 i} H_{2} R_{k}}{P_{r j} H R_{1 k}+\frac{p_{1 i}\left|h_{1} r_{k}\right|^{2}+p_{2 i}\left|h_{2} r_{k}\right|^{2}+\left(n r_{i}^{k}\right)^{2}}{n r_{i}^{k^{2}}}}
\end{gathered}
$$

Then,

$$
S I N R_{1}=\frac{P_{r j} H R_{1 k} P_{2 i} H_{2} R_{k}}{P_{r j} H R_{1 k}+p_{1 i} H_{1} R_{k}+p_{2 i} H_{2} R_{k}+1}
$$




$$
\begin{gathered}
\operatorname{SINR}_{2}=\frac{\frac{A_{f}^{2}}{A_{f}^{2}} p_{r j} \frac{\left|h r_{2 k}\right|^{2}}{n_{2 j}^{k^{2}}} p_{1 i} \frac{\left|h_{1} r_{k}\right|^{2}}{n r_{i}^{k^{2}}}}{\frac{A_{f}^{2}}{A_{f}{ }^{2}} p_{r j} \frac{\left|h r_{2 k}\right|^{2}}{n_{2 j}^{k^{2}}} \frac{n r_{i}^{k^{2}}}{n r_{i}^{k^{2}}}+\frac{n_{2 j}^{k^{2}}}{A_{f}{ }^{2} n_{2 j}^{k^{2}} n r_{i}^{k^{2}}}} \\
=\frac{P_{r j} H R_{2 k} P_{1 i} H_{1} R_{k}}{P_{r j} H R_{2 k}+\frac{p_{1 i}\left|h_{1} r_{k}\right|^{2}+p_{2 i}\left|h_{2} r_{k}\right|^{2}+\left(n r_{i}^{k}\right)^{2}}{n r_{i}^{k^{2}}}}
\end{gathered}
$$

and,

$$
S I N R_{2}=\frac{P_{r j} H R_{2 k} P_{1 i} H_{1} R_{k}}{P_{r j} H R_{2 k}+p_{1 i} H_{1} R_{k}+p_{2 i} H_{2} R_{k}+1}
$$

$$
\text { where, } H_{n} R_{k}=\frac{\left|h_{n} r_{k}\right|^{2}}{n r_{i}^{k^{2}}}, H R_{1 k}=\frac{\left|h r_{n k}\right|^{2}}{n_{1 j}^{k^{2}}}
$$

where, $S I N R_{1}, S I N R_{2}$ are signals to noise plus cross-tier interference ratio calculated at $S U_{1}, S U_{2}$, respectively after the signal is amplified and broadcasted from $k$ relay on subcarrier $j$.

The total transmission rate of SUs with the assistance of AF with two-way protocol of $k^{\text {th }}$ relay on subcarrier pairs $\langle i, j\rangle$ can be calculated with two time slots to complete the transmission messages between $S U_{s}:[15]-[18]$.

$$
R_{A F}^{i, j, k}=\frac{1}{2}\left(\log _{2}\left(1+S I N R_{1}\right)+\log _{2}\left(1+S I N R_{2}\right)\right)
$$

\section{B. Interference Analysis for Secondary Network}

To analyze the interference from secondary network (SUs with relay nodes) to primary network with a perfect sensing of licensed spectrum, means that the SUs detect only the unused subcarrier perfectly, leading to SUs use subcarriers that are different from primary users. The interference power from secondary users on subcarriers $i$, when sending to all $k^{\text {th }}$ relay nodes in MA phase denote as $I_{s i}$, and the interference power from $k^{\text {th }}$ relay on subcarrier $j$, when they amplify and broadcast to SUs in BC phase denote as $I_{r j}$, and must be less than $I_{t h}$ threshold interference defined by Primary network.

$$
\begin{aligned}
& I_{s i}=\sum_{i \in N_{S}} \sum_{k \in \mathcal{K}}\left(p_{1 i} \sum_{l \in N_{p}}\left|f_{1 l}\right|^{2}+p_{2 i} \sum_{l \in N_{p}}\left|f_{2 l}\right|^{2}\right) \\
& I_{r j}=\sum_{j \in N_{s}} \sum_{k \in \mathcal{K}}\left(p_{r j} \sum_{l \in N_{p}}\left|f_{r l}\right|^{2}\right) \\
& \leq I_{t h}
\end{aligned}
$$

To analyze the cross-tier interference from MBS to secondary network, the cross-tier interference power affect on $k^{\text {th }}$ relay when it receives a signals from SUs in MA on sub-carrier $i$ is $Z_{i}^{k}$ calculated in (17). The cross-tier interference power affect on SUs receiving signals from $k$ relay in $\mathrm{BC}$ on sub-carrier $j$ is $Z_{1 j}^{k}$ and $Z_{2 j}^{k}$ calculated in (18).

$$
\begin{array}{r}
z_{i}^{k} \\
=\sum_{l \in N_{p}}\left(\sum_{k \in \mathcal{K}} p_{M B S} \sum_{i \in N_{S}}\left|h c_{k i}\right|^{2}\right) \\
Z_{s j}^{k}=\sum_{l \in N_{p}} \sum_{k \in \mathcal{K}}\left(p_{M B S} \sum_{j \in N_{S}}\left|h c_{1 j}\right|^{2}\right. \\
\left.+p_{M B S} \sum_{j \in N_{S}}\left|h c_{2 j}\right|^{2}\right)
\end{array}
$$

\section{PROBLEM STATEMENT (OBJECTIVE FUNCTION)}

The identification of the objective function in this model is to maximize the total data rate for secondary network, with the power constraints for secondary network and the interference constraints from secondary network to macro cell network. To solve this problem, a two dimension binary matrix $A_{i \times j}$ is considered. Where $A \in\{0,1\}$, if $\mathrm{A}=1$, means that the subcarrier $i$ is allocated to SUs when transmit to $k^{t h}$ relay on MA phase. And the subcarrier $j$ is allocated to $k^{\text {th }}$ relay when AF the signal and transmit to SUs on BC phase. Then the subcarrier $i$ is paired with subcarrier $j$ denoted by a symbol $\langle i, j\rangle$. In addition, a three dimension matrix $B_{i \times j \times k}$ is considered, where $B \in\{0,1\}$ if $\mathrm{B}=1$ means that the subcarrier pair $\langle i, j\rangle$ allocated for $k^{t h}$ relay. To solving this formulation problem to maximization the total transmission rate for some constraints. The Power constraint from SUs is c3 and for $k^{t h}$ relay is $\mathrm{c} 4$. The interference constraint from SUs is c5 and from $k$ relay is c6 [15], [19], [20].

$$
\begin{aligned}
& O P:\left(\begin{array}{c}
\max \\
A, B, P
\end{array}\right) \quad \sum_{i \in N_{S}} \sum_{j \in N_{S}} \sum_{k \in \mathcal{K}} B_{i, j}^{k} A_{i, j} R_{A F}^{i, j, k} \\
& \text { c1: } \sum_{i \in N_{S}} A \\
& =1, \sum_{j \in N_{S}} A \\
& =1 \\
& \text { c2: } \sum_{k \in \mathcal{K}} B=1, \text { for all }<i, j \\
& \text { c3: } \sum_{i \in N_{s}} \sum_{k \in \mathcal{K}} p_{s i} \leq p_{\text {smax }} \text { for } s \\
& =1,2
\end{aligned}
$$


Al-Furat Journal of Innovation in Electronics and Computer Engineering (FJIECE) is an international, open access, interdisciplinary, quarterly, and double blind peer-reviewed journal published by Al-Furat Al-Awsa Technical University/Iraq. It is dedicated to the latest advancement in Electronic Engineering, Computer engineering, and their related and subfields.

$$
\begin{aligned}
& c 4: \sum_{j \in N_{S}} p_{r j} \\
& \begin{array}{l}
\leq p_{r \text { max }} \text { for all } k \\
c 5: I_{s i}=\sum_{i \in N_{s}} \sum_{k \in K}\left(p_{1 i} \sum_{\substack{l \in N_{p} \\
\leq}}\left|f_{1 l}\right|^{2}+p_{2 i} \sum_{l \in N_{p}}\left|f_{2 l}\right|^{2}\right)
\end{array}
\end{aligned}
$$

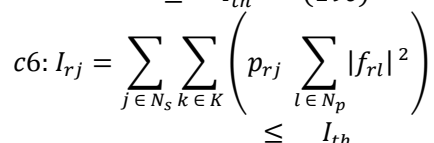

\section{SIMULATION RESULTS}

The simulation results obtained the power allocation and specific number of subcarriers allocation for small cell users and for relay nodes, under power constraints and interference threshold constraints to avoid the interference from small cells to macro cell because use the same frequency band, with the consideration of cross-tier interference. Particularly this section will find the optimized power allocated for the cognitive small cell users $S U_{S}$ and relay nodes, and sub-carrier pairing matrix to the maximization of the total data rate evaluated in numerical results of the implemented system model. Assuming the maximum transmission power of all elements ( $p_{s \text { max }}$, $p_{\text {rmax }}$, and $\left.p_{M B S}\right)$ be in normalized power that is equal to 1 watt, the adopted all channel gains and interferencechannel gains are i.i.d as $\mathcal{C N} \sim\left(\mu, \sigma^{2}\right)$ with zero mean [15].

All parameters for GA and the powers values used in this system model listed in Table-II.

TABLE-II: GA PARAMETERS AND POWER VALUES

\begin{tabular}{ll}
\hline number of iterations size data & $\mathbf{5 0}$ \\
\hline number of initial generations data & 200 \\
\hline total number of variables & $\left(X_{T}=48\right)$ \\
\hline power variables & $\begin{array}{l}\left(X_{P}=24\right), \quad \text { its } \\
\text { range } 256 \text { levels }\end{array}$ \\
\hline subcarrier variables & $\begin{array}{l}\left(X_{F}=24\right), \text { binary } \\
\text { range }(0 \text { or } 1)\end{array}$ \\
\hline $\begin{array}{l}\text { Maximum transmission power for } \\
\text { secondary users }\end{array}$ & $p_{\text {s max }}=1 \mathrm{watt}$ \\
\hline $\begin{array}{l}\text { Maximum transmission power for } \\
\text { relay nodes }\end{array}$ & $p_{\text {rmax }}=1 \mathrm{watt}$ \\
\hline $\begin{array}{l}\text { Maximum transmission power for } \\
\text { macro cell }\end{array}$ & $p_{M B S}=1 \mathrm{watt}$ \\
\hline interference power threshold & $1 \times 10^{-9}$ watt \\
\hline AWGN & zero-mean and \\
& 0.01 variance \\
\hline
\end{tabular}

This is to guarantee the inter-carrier-interference that does not occur from carrier overlapping, assuming an offset constant randomly is between $(0$ and 1$)$ for each subcarrier $i$ and $j$ [21].

The results for this system model:
1. The optimized sub-carrier pairing matrix and relay selection matrix for relay1 and relay 2 are $\mathrm{A}, \mathrm{B} 1$, and $\mathrm{B} 2$ respectively:

$$
\begin{gathered}
A=\left[\begin{array}{llll}
1 & 0 & 0 & 0 \\
0 & 0 & 1 & 0 \\
0 & 0 & 0 & 1 \\
0 & 1 & 0 & 0
\end{array}\right] \\
B 1=\left[\begin{array}{llll}
0 & 1 & 0 & 1 \\
0 & 1 & 0 & 1 \\
0 & 1 & 0 & 1 \\
0 & 1 & 0 & 1
\end{array}\right], \quad B 2=\left[\begin{array}{llll}
1 & 0 & 1 & 0 \\
1 & 0 & 1 & 0 \\
1 & 0 & 1 & 0 \\
1 & 0 & 1 & 0
\end{array}\right] \\
A \times B 1=\left[\begin{array}{llll}
0 & 0 & 0 & 0 \\
0 & 0 & 0 & 0 \\
0 & 0 & 0 & 1 \\
0 & 1 & 0 & 0
\end{array}\right], \quad A \times B 2=\left[\begin{array}{llll}
1 & 0 & 0 & 0 \\
0 & 0 & 1 & 0 \\
0 & 0 & 0 & 0 \\
0 & 0 & 0 & 0
\end{array}\right]
\end{gathered}
$$

Each number in matrix $A$ represents the $\langle i, j\rangle$ pair. The rows represent the subcarrier $i=\left[\begin{array}{llll}1 & 2 & 3 & 4\end{array}\right]$ and the columns represent the subcarrier $j=\left[\begin{array}{llll}1 & 2 & 3 & 4\end{array}\right]$, when $\mathrm{A}=1$ in the first row, meaning that the user uses the subcarrier $(i=1)$ for sending to the $k^{\text {th }}$ relay (of MA phase) and the $k^{\text {th }}$ relay uses the subcarrier $(j=1)$ for forwarding to user (of BC phase). The B1, B2 are the relay assignment matrix depending on matrix $A$. To determines which subcarrier pairs are assignment to $R_{1}$ or $R_{2}$ will be multiply matrix $A$ by matrices $B 2$, and $B 1$, (dot product) respectively. That meaning, the $\langle 4,2\rangle$ pair is assigned to relay $1,<3,4\rangle$ pair is assigned to relay $1,<1,1\rangle$ pair is assigned to relay 2 , and $<2,3\rangle$ pair is assigned to relay2. Figure 3 illustrates the subcarrier pair allocated for $S U_{1}$ with each relay and for $S U_{2}$ with each relay.
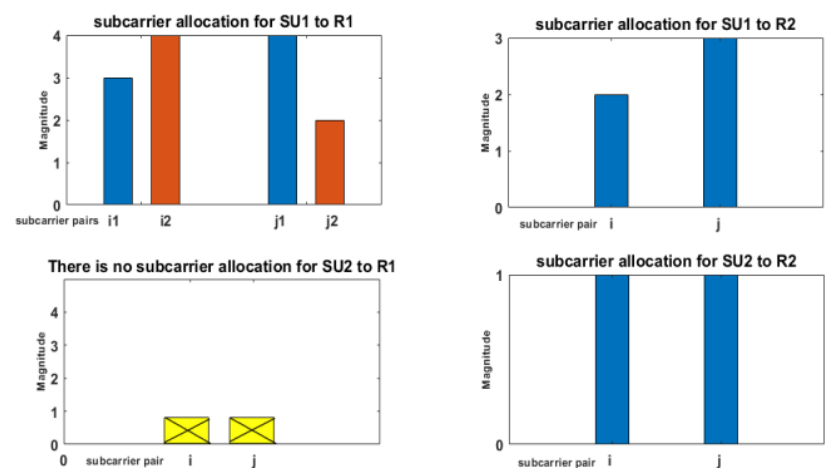

Fig.3: Subcarrier Allocation for $S U_{s}$ with each relay

2. The powers allocated for $S U_{s}$ and Relay nodes shown in Fig. 4 and 5 respectively. Figure 4 shows the transmit power allocation for MA phase from SUs on all subcarriers $i$ to the relay nodes under the maximum power constraint which is equal to normalized power $p_{s \max }=1$ watt. Figure 5 shows the power allocation from the relay nodes $R_{1}$ and $R_{2}$ for $\mathrm{BC}$ phase on all subcarriers $j$ to $S U_{S}$ under the maximum power constraint which is equal to normalized power $p_{r \max }=1$. From Fig.4 and Fig.5, the summation of 
Al-Furat Journal of Innovation in Electronics and Computer Engineering (FJIECE) is an international, open access, interdisciplinary, quarterly, and double blind peer-reviewed journal published by Al-Furat Al-Awsat Technical University/Iraq. It is dedicated to the latest advancement in Electronic Engineering, Computer engineering, and their related and subfields.
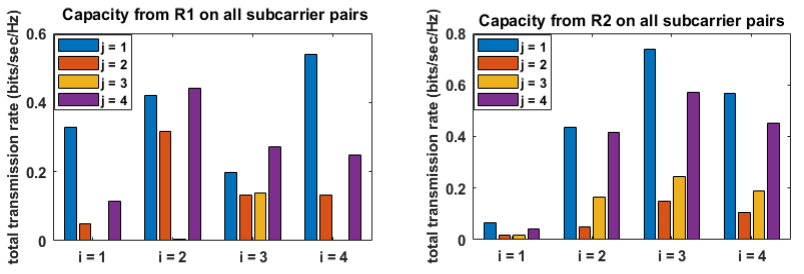

Fig.7: The maximum capacity for SUs with relay nodes R1 and R2 under maximum power constraint and interference threshold

The total data rate of this system model it is the result of adding the two rates: Total transmission data rate $=$ $5.0686 \mathrm{bit} / \mathrm{s} / \mathrm{Hz}$. From this result, the decreasing in the interference threshold will help in increasing the total transmission data rate of this system model.

\section{CONCLUSION AND DISCUSSION}

In this paper, the joint resources allocation RA consist of power allocation, sub-carrier pairing allocation and relay selection have proposed, under the power constraint and interference power constraint to improve the total system capacity of cognitive small cell HetNet in a perfect spectrum sensing. The cross-tier interference considered from macro cell (primary network) to small cells and AF two-way relay nodes (secondary network). The genetic algorithm GA used to solve the optimization problems to maximize the total transmission data rate of cognitive secondary network within macro cell. The results have showed the ability of GA to effectively determine the frequency and power values of secondary network (small cell users and relay nodes) without interference with the primary network (all problem formulation are solved under all constraints leading to increasing in the spectrum efficiency by exploiting the vacant band. Can be getting higher transmission data rate when the interference threshold decreased, that meaning the interference constraint will be more flexible and allow the SUs from achieve their transmission rate better, because the SINR will be increased if interference will decreased and it lead to increasing in the capacity and finally increasing the total transmission rate.

\section{REFERENCES}

[1] Prof. Simon R. Saunders, Indoor Wireless Communications: From Theory to Implementation, Small cell (chapter 10), $1^{\text {st }}$ ed., Wiley Telecom, (2017)

[2] Gamage, Amila Tharaperiya, and Xuemin (Sherman) Shen, Resource management for heterogeneous wireless networks, Springer International Publishing, (2018).

[3] Grace, David, and Honggang Zhang, Distributed Artificial Intelligence (DAI), Regulatory Policy and Economics, Implementation, Wiley, New York, NY, USA, (2012).

[4] Liang, Liang, Gang Feng, and Yunjian Jia, Game-theoretic hierarchical resource allocation for heterogeneous relay network,
IEEE Transactions on Vehicular Technology 64.4 (2014): 14801492.

[5] Chen, Zhengchuan, et al, Cooperation in 5G heterogeneous networking: Relay scheme combination and resource allocation, IEEE Transactions on Communications 64.8 (2016) 3430-3443.

[6] Patra, Tapas Kumar, and Albert Sunny, Forwarding in heterogeneous mobile opportunistic networks, IEEE Communications Letters 22.3 (2018): 626-629.

[7] Xu, Yongjun, et al, Optimal power allocation for multiuser OFDMbased cognitive heterogeneous networks, China Communications 14.9 (2017): 52-61.

[8] $\mathrm{Li}, \mathrm{Li}$, Changqing $\mathrm{Xu}$, and Meixia Tao, Resource allocation in open access OFDMA femtocell networks, IEEE Wireless Communications Letters 1.6 (2012): 625-628.

[9] Zhang, Lei, Tao Jiang, and Kai Luo, Dynamic spectrum allocation for the downlink of OFDMA-based hybrid-access cognitive femtocell networks, IEEE Transactions on Vehicular Technology 65.3 (2015): 1772-1781.

[10] Park, Hyunsung, and Taewon Hwang, Energy-efficient power control of cognitive femto users for $5 G$ communications, IEEE Journal on Selected Areas in Communications 34.4 (2016): 772 785.

[11] LeAnh, Tuan, et al, Distributed power and channel allocation for cognitive femtocell network using a coalitional game in partitionform approach, IEEE Transactions on Vehicular Technology 66.4 (2016): 3475-3490.

[12] Liu, Zhixin, et al, Power Allocation Based on ProportionalIntegral Controller in Femtocell Networks With Consideration of Maximum Power Constraint, IEEE Systems Journal 13.1 (2018): 88-97.

[13] Rodríguez, Leonardo Jiménez, Nghi Tran, and Tho LeNgoc, Amplify-and-Forward Relaying in Wireless Communications, Springer, 2015.

[14] Alsharoa, Ahmad, Faouzi Bader, and Mohamed-Slim Alouini, Relay selection and resource allocation for two-way DF-AF cognitive radio networks, IEEE Wireless Communications Letters 2.4 (2013): 427-430.

[15] Li, Hangqi, and Xiaohui Zhao, Joint resource allocation for OFDM-based cognitive two-way multiple AF relays networks with imperfect spectrum sensing, IEEE Transactions on Vehicular Technology 67.7 (2018): 6286-6300.

[16] Chen, Chang-Lin, and Che Lin, Utility maximization for two-way AF relaying under rate outage constraints, IEEE Transactions on Wireless Communications 16.9 (2017): 5762-5778.

[17] Ji, Xiaodong, et al, On the study of half-duplex asymmetric twoway relay transmission using an amplify-and-forward relay, IEEE Transactions on Vehicular Technology 61.4 (2012): 1649-1664.

[18] Wang, Yurong, et al, Hybrid one-way full-duplex/two-way halfduplex relaying scheme, IEEE Access 5 (2017): 7737-7745.

[19] Shaat, Musbah, Telex MN Ngatched, and Octavia A. Dobre, Resource allocation in OFDM-based cognitive two-way multiplerelay networks, 2014 IEEE Global Communications Conference. IEEE, 2014

[20] Lang, Hung-Sheng, Shih-Chun Lin, and Wen-Hsien Fang, Subcarrier pairing and power allocation with interference management in cognitive relay networks based on genetic algorithms, IEEE Transactions on Vehicular Technology 65.9 (2015): 7051-7063.

[21] Ma, Tianming, A new inter-carrier interference self-cancellation mapping scheme over fast time-varying fading channels, Electronics Letters 53.21 (2017): 1433-1435. 